\title{
A High Resolution Dynamic Heart Model Based on Averaged MRI Data
}

\author{
John Moore ${ }^{1,2}$, Maria Drangova ${ }^{1}$, Marcin Wierzbicki ${ }^{1}$, John Barron ${ }^{2}$, and \\ Terry Peters ${ }^{1}$ \\ 1 Robarts Research Institute and University of Western Ontario \\ London, Ontario, Canada N6A 5K8 \\ 2 Dept. of Computer Science \\ University of Western Ontario \\ London, Ontario, Canada N6A 5B7 \\ \{jmoore, mdrangov, mwierz, tpeters\}@imaging.robarts.ca
}

\begin{abstract}
We are in the process of constructing a high resolution, high signal to noise ratio (SNR) dynamic MRI dataset for the human heart using methodology similar to that employed to construct a low-noise standard brain at the Montreal Neurological Institute. Several high resolution, low SNR magnetic resonance images of 20 phases over the cardiac cycle were acquired from a single subject. Images from identical phases and temporally adjacent phases were registered, and the image intensities were averaged together to generate a high resolution, high SNR dynamic magnetic resonance image volume of the human heart. Although this work is still preliminary, and the results still demonstrate residual artifacts due to motion an sub-optimal alignment of interleaved image slices, our model has a SNR that is improved by a factor of 2.7 over a single volume, spatial resolution of $1.5 \mathrm{~mm}^{3}$, and a temporal resolution of 60 ms.
\end{abstract}

\section{Introduction}

Magnetic resonance imaging (MRI) involves a compromise between spatial resolution, signal to noise ratio (SNR), and acquisition time, among other factors. In the case of cardiac MRI, this compromise is further complicated by both heart motion and the fact that the images are typically acquired under breathhold conditions. This compromise usually results in images with high in-plane resolution in two dimensions, but anywhere from $6 \mathrm{~mm}$ to $10 \mathrm{~mm}$ thick slices. Although super-high resolution may not be required for diagnosing many cardiac diseases, image guided surgery (IGS) would benefit from high resolution isotropic 3D images. The application of IGS to neurosurgery has benefited from the availability of a high resolution brain model [1/2]. Since heart surgery often requires a level of precision similar to that required for neurosurgery, we believe that cardiac IGS [3] can benefit from a high resolution, high SNR dynamic heart model.

Linear or higher order interpolation is traditionally used to re-sample a nonisotropically sampled volume, to one that is sampled uniformly in all directions 
[4.5]. Interpolation algorithms can infer general changes in intensity, but they inevitably blur detail such as valvular function and wall motion, which could be important in IGS. In addition, it is difficult to assess the efficiency of such procedures without a high resolution "gold standard" image with which to compare the results.

In this paper, we report on a technique to generate a high SNR MR heart model with $1.5 \mathrm{~mm}$ isotropic voxels. The model is based on a large number of high resolution images of one subject, registered and intensity-averaged to improve SNR. The model is still under construction with more image sets currently being collected, and at least 12 complete 4D series planned, in addition to a number of miscellaneous complementary images. As this dataset evolves, it will be possible to use it in a manner similar to the "average brain" developed at the Montreal Neurological Institute, which has found extensive use as both a standard model and a repository for anatomical and electro-physiological atlas data 6. The heart model will be available as a research tool in the development of cardiac IGS, and will also provide data that can be used to validate techniques such as interpolation and segmentation procedures. Eventually, several models will be constructed, to represent variations in heart morphology.

\section{Method}

\subsection{Image Acquisition}

Images of a healthy volunteer were acquired on a 1.5 T GE CVi scanner (GE medical systems, Milwaukee). Each complete 3D MR image consisted of 75 coronal slices $(1.5 \mathrm{~mm}$ thick,) with an in-plane resolution of $1.5 \mathrm{~mm}$. A single imaging session consisted of acquiring twenty prospectively gated, segmented gradient echo T1-weighted images at each slice location with the following scan sequence specifications: $256 \times 128$ image matrix; 8 views per segment; 4 signal averages (NEX); flip angle 20 degrees. Breath-holds were 42 seconds in duration. Each acquisition was interleaved so that every third slice was acquired in sequence. The time to acquire a complete $4 \mathrm{D}$ representation of the cardiac cycle was approximately 2.5 hours. Coronal images were acquired since this minimized the number of slices needed to cover the entire heart, thus minimizing scan time. To date, four complete 4D series, each consisting of $203 \mathrm{D}$ volumes, have been acquired.

\subsection{Image Processing}

Because the image slices exhibit high resolution in all three dimensions, two major obstacles must be dealt with; image misalignment due to independent breath-holds, and relatively low SNR. Slight variations in lung volume in different breath-holds result in misalignment of the coronal images, when a $3 \mathrm{D}$ volume is viewed in the sagittal or axial plane (see Figure 1a). The maximum observed shift between coronal slices was $9 \mathrm{~mm}$ in the superior-inferior (SI) direction, 
based on the location of the diaphragm and $4.5 \mathrm{~mm}$ in the left-right (LR) direction, based on the location of the ventricular septum. Variations in lung volume between breath-holds can also cause tissue motion in the anterior-posterior (AP) direction, but since the images were acquired in the coronal plane, tissue movement in the AP direction result either an under- or over-sampling of data in a particular part of the volume, (i.e., because of differences in inspiration volume between breath-holds, nominally adjacent slices may sample the same slice of the volume.)
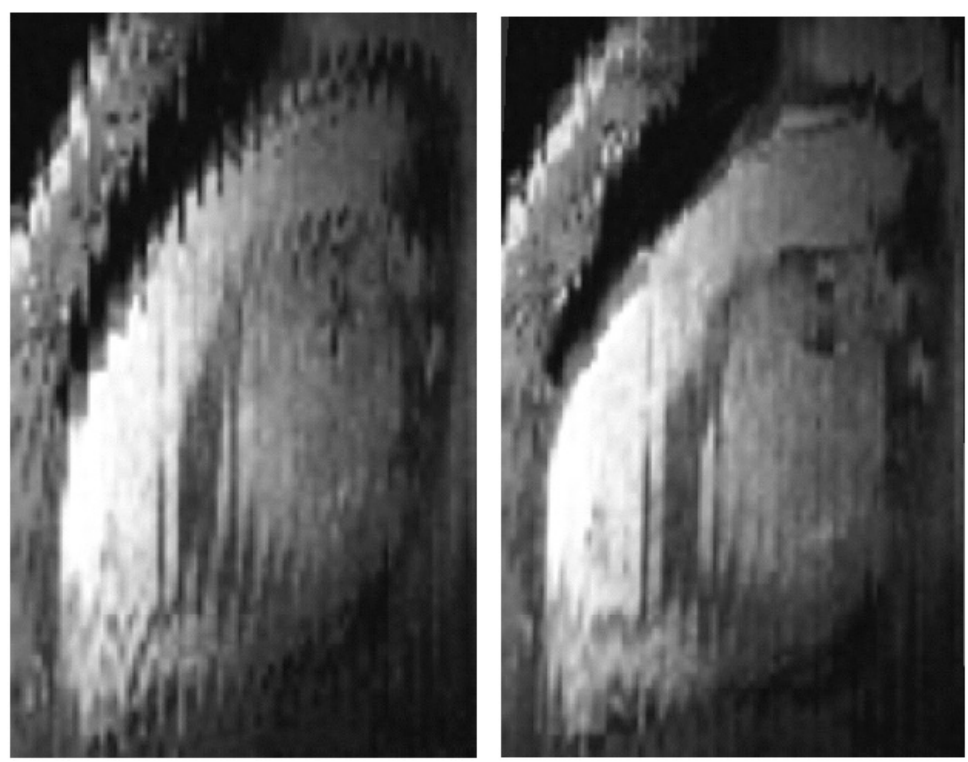

(a) Before misalignment correction (b) After misalignment correction

Fig. 1. Sagittal slice from a 3D cardiac image at end diastole before and after misalignment correction.

Breath-Hold Related Misalignment. Breath-hold related misalignments are minimized by using high resolution isotropic single slice images acquired sagittally and axially and employed as "ground truth" representing the expected appearance of the 3D volume in these planes. Since most motion caused by breathing variations occurs in the superior-inferior (SI) direction [78], the sagittal slice was registered first, using rigid body registration, to the appropriate location in the 3D volume. A line-by-line mean squared difference (MSD) best fit in the SI direction was computed. The resulting transformation was then applied to each slice in the full 3D volume. The process was then repeated using the "ground truth" axial slice, but with tighter constraints on the maximum LR shift allowed. Breath-hold related inaccuracies in the AP axis could not be corrected in this manner, since signal in this dimension was either lost or duplicated, as discussed earlier. The averaging process provided the only compensation possible in this dimension. 
Image Averaging. After correcting for breath-hold misalignments, the SNR was improved by a combination of image registration and image intensity averaging. An elastic image registration algorithm developed in our laboratory [9] was used for this purpose. The acquisition of multiple MRI volumes of the heart throughout the cardiac cycle allows unique opportunities when using the averaging process to improve SNR. For example, images from different phases can be registered ("temporal registration") or images representing the same cardiac phase, but acquired during different sessions can be registered ("atemporal registration"). Both of these strategies were employed in our procedure.

The registration and averaging strategy was based on Guimond 10] and Frangi [1]. In their work, one image from the pool of images to be averaged is randomly selected to be the target. All other images are then registered to the target image, and the image intensities are averaged together. In order to minimize any bias caused by the choice of initial target image, the averaged image is then used as the target, with all source images being registered to this average volume. The process is iterated until a sufficient level of similarity is achieved between the input and output averaged images. Given the coronal slice misalignment caused by breath-hold variations, this process was modified slightly as follows.

Let $I_{k}^{j}$ represent a 3D cardiac image, where superscript ' $j$ ' represents the image acquisition session, while ' $k$ ' represents the cardiac phase of the $3 \mathrm{D}$ volume. For a given image acquisition session, all 20 phases for a coronal slice are acquired during the same breath-hold. Thus, for example, the breath-hold related misalignments in $I_{20}^{1}$ are identical to those in $I_{19}^{1}$, and so on. As a result, any misalignments remaining after the breath-hold correction method explained previously would not be averaged out after temporal registration. Consequently, a preliminary model was constructed using only atemporal registration. Images $I_{20}^{1}, I_{20}^{3}$, and $I_{20}^{4}$ were registered to $I_{20}^{2}$. The registered images were averaged to form the atemporal averaged model.

The atemporally registered model minimized the presence of any misalignments which remained after breath-hold correction. In order to further increase the factor by which SNR was improved, images of temporally adjacent phases, as well as images representing the same phase in the cardiac cycle, were then registered to this preliminary model. The resulting images were then averaged together. This process was iterated to achieve an acceptable level of convergence. In total, atemporal averaging was based on 4 images per phase. Combined with temporally adjacent phases, this yielded a total of 12 images averaged together for each phase. Using this approach, SNR should be increased by a factor of $\sqrt{12}$ or 3.46. As was mentioned earlier, a total of at least 12 complete $4 \mathrm{D}$ series are planned, which will effectively generate 36 images available to be averaged, resulting in an improvement in SNR by a factor of 6 .

\subsection{SNR Measurement}

Signal was measured from the left ventricular cavity. Since regions outside the chest were not imaged, noise was measured from a region inside the lungs. Since 
noise was measured in essentially a no-signal region where noise is rician rather than gaussian, SNR was calculated based on Henkelman [12] and Kaufman [13] where the factor of 1.5 is included to account for underestimation of the noise standard deviation when measured in air in a magnitude image:

$$
\mathbf{S N R}=\frac{\text { mean }(\text { signal })}{1.5 \times \operatorname{stddev}(\text { noise })} .
$$

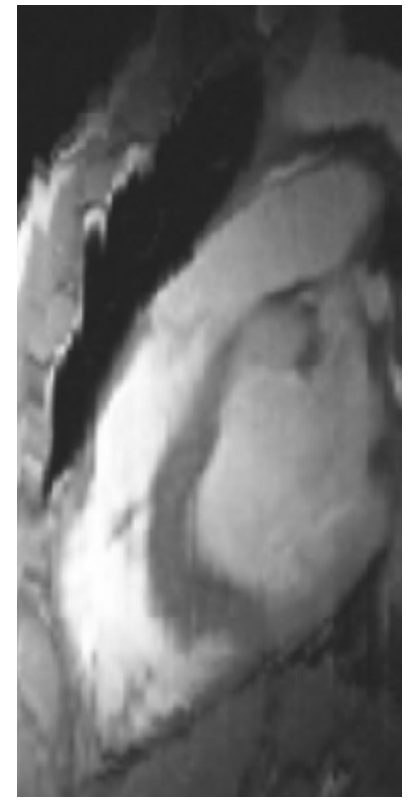

(a) sagittal view

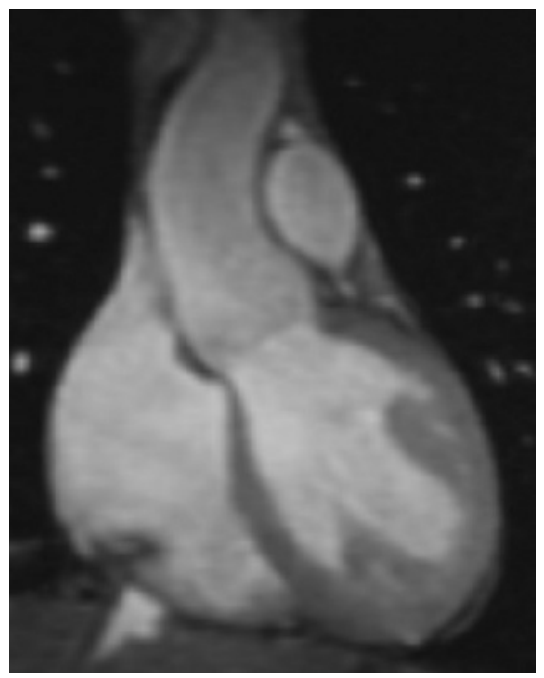

(b) coronal view

Fig. 2. The sagittal and coronal views of the model at end diastole, based on intensity averaging of twelve $3 \mathrm{D}$ volumes.

\section{Results and Discussion}

Our breath-hold misalignment correction technique was effective in aligning the coronal slices along the boundary defined by the pulmonary artery. Some misalignment remained, particularly near the apex of the heart along the diaphragm, where a shift of between $1 \mathrm{~mm}$ and $3 \mathrm{~mm}$ could be observed in the sagittal plane. This suggests the heart undergoes a slight change in shape, and not simply a change in location as a result of different breath-holds. It should also be noted that the first data series (shown in Figure 1a) contained the poorest alignment; by comparison subsequent series have required very little correction. 


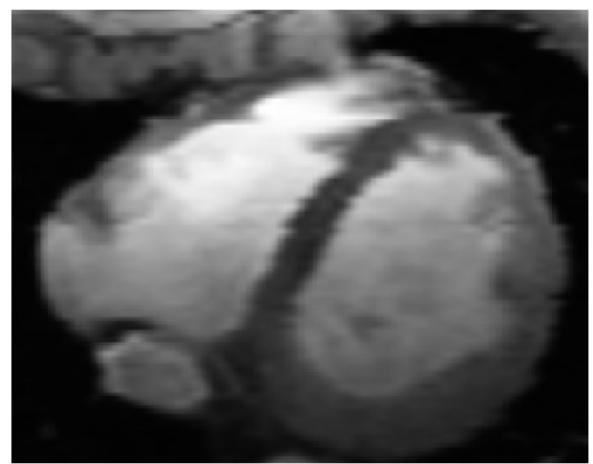

(a) axial view

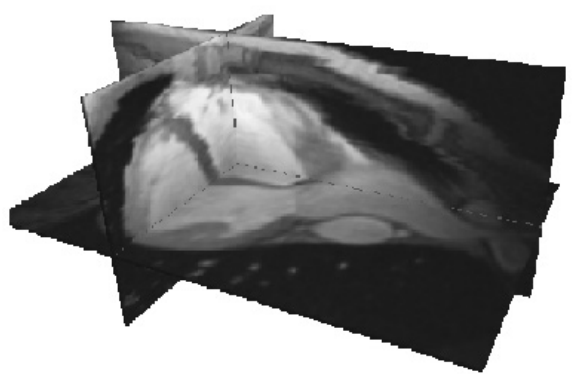

(b) oblique view

Fig. 3. Axial view and a sample oblique view of the model at end diastole, based on intensity averaging of twelve $3 \mathrm{D}$ volumes.

Table 1. Changes in SNR for images acquired at end diastole.

\begin{tabular}{|l|c|c|}
\hline Image & SNR & Factor of improvement \\
\hline$I_{20}^{1}$ & 26.0 & 0 \\
\hline$A_{20}^{1}$ & 51.6 & 1.98 \\
\hline Final Model & 70.7 & 2.7 \\
\hline
\end{tabular}

A comparison of Figure 1 1 with Figure $2 \mathrm{a}$ provides a clear visual example of how intensity averaging can improve SNR as well as detail. The SNR in the raw $3 \mathrm{D}$ volumes for the end-diastole images ranged from 31.3 to 24.7 . The atemporal average model improved SNR by a factor of 1.98 (Table 1 ); the expected result for averaging 4 images was 2. The factor of SNR improvement for the 12-image averaged model was 2.7. Averaging 12 images together produced slightly less improvement than was expected. Improved breath hold correction techniques and further modifications to the elastic registration algorithm may yield higher SNR values. At present, our averaging algorithm applies only to the image intensities; computing an average shape, as outlined in Guimond [10] and Frangi [1], may also improve the overall results.

Acknowledgments. Mark Wachowiak and Ravi Gupta, Robarts Research Institute, assisted in preparation and editing of the manuscript. We would also like to thank Atamai, Inc. for software and code development.

Funding for this project has been provided by the following sources: Canadian Institute of Health Research MT 14735, Ontario Consortium for Image-guided Surgery and Therapy, Heart and Stroke Foundation of Canada grant NA 4755 and an NSERC operating grant. M. Drangova is supported by a scholarship from the CIHR, and M. Wierzbicki acknowledges funding from NSERC. 


\section{References}

1. Finnis, K.W., Starreveld, Y.P., Parrent, A.G., Sadikot, A.F., Peters, T.M.: 3D functional database of subcortical structures for surgical guidance in image guided neurosurgery. MICCAI-99 (1999) 758-767

2. Finnis, K.W., Starreveld, Y.P., Parrent, A.G., Peters, T.M.: A 3D database of deep brain functional anatomy and its application to image guided neurosurgery. MICCAI-2000 (2000) 1-8

3. Chiu, A., Dey, D., Drangova, M., Boyd, W.D., Peters, T.M.: 3D image guidance for Minimally Invasive Robotic Coronary Artery Bypass (MIRCAB). Heart Surgery Forum, 3(3): (2000) 224-231

4. Raya, P.S., Udupa,J.K.: Shape-based interpolation of multidimensional objects. IEEE Transactions on Medical Imaging 9 (1990) 32-42

5. Herman, G.T., Zheng, J., Bucholtz, C.A.: Shape-based interpolation. IEEE Computer Graphics and Applications. (1992) 69-79

6. Holmes, C., Hoge, R., Collins, L., Woods, R., Toga, A., Evans, A.: Enhancement of MR images using registration for signal averaging. Journal of Computer Assisted Tomography 22 (1998) 324-333

7. McLeish, K., Hill, D., Atkinson,D., Blackall, J., Razavi, R.: A study of the motion and deformation of the heart due to respiration. IEEE Trans. Medical Imaging 21 (2002)1142-1150

8. Pai, V.M., Wen, H.: Isolating the cardiac blood pool position for use as a marker of heart position in free-breathing MRI examinations. IEEE Conf. Medical Imaging (2002) 939-942

9. Wierzbicki, M., Peters, T.M.: Determining epicardial surface motion using elastic registration:towards virtual reality guidance of minimally invasive cardiac interventions. MICCAI-2003 (2003)

10. Guimond, A., Meunier, J., Thirion, J.-P.: Average brain models: a convergence study. Computer Vision and Image Understanding 77 (2000) 192-210

11. Frangi, A.F., Rueckert, D., Schnabel, J.A., Niessen, W.J.: Automatic 3D ASM construction via atlas-based landmarking and volumetric elastic registration. Information Processing in Medical Imaging. 17th International Conference, IPMI (2001) 78-91

12. Henkelman, R.M.: Measurement of signal intensities in the presence of noise in MR images. Med. Phys. 12 (1985) 232-233

13. Kaufman, L., Kramer, D., Crooks, L., Ortendahl, D.: Measuring signal-to-noise ratios in MR imaging. Radiology (1989) 265-267 